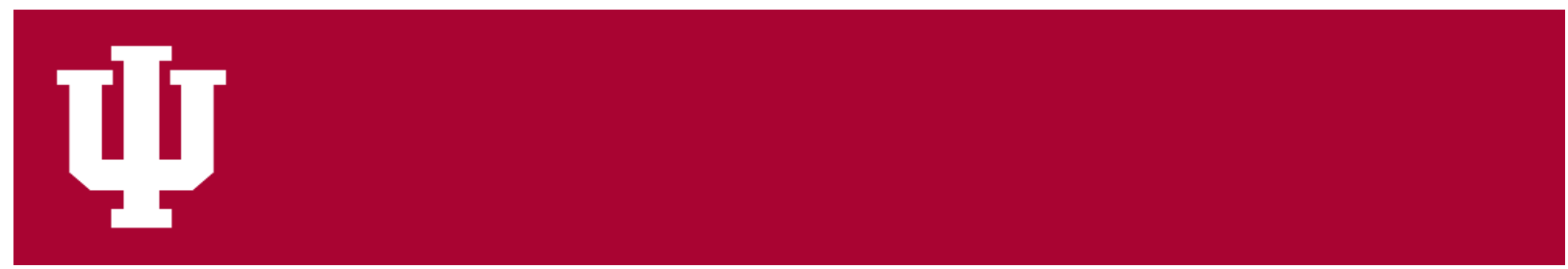

IUScholarWorks at Indiana University South Bend

\title{
Bernstein Polynomial Model for \\ Nonparametric Multivariate Density
}

Wang, Tao; Guan, Zhong

This is an Accepted Manuscript of an article published by Taylor \& Francis in

Statistics on Feb.06, 2019, available online:

https://doi.org/10.1080/02331888.2019.1574299.

This document has been made available through IUScholarWorks repository, a service of the Indiana University Libraries. Copyrights on documents in IUScholarWorks are held by their respective rights holder(s). Contact iusw@indiana.edu for more information. 


\title{
Bernstein Polynomial Model for Nonparametric Multivariate Density
}

\author{
Tao Wang ${ }^{\mathrm{a}}$ and Zhong Guan ${ }^{\mathrm{b}}$
}

${ }^{a}$ School of Mathematical Sciences, Harbin Normal University, Harbin, China; ${ }^{b}$ Department of Mathematical Sciences, Indiana University South Bend, South Bend, Indiana, USA

\author{
ARTICLE HISTORY \\ Compiled September 14, 2018
}

\begin{abstract}
In this paper, we study the Bernstein polynomial model for estimating the multivariate distribution functions and densities with bounded support. As a mixture model of multivariate beta distributions, the maximum (approximate) likelihood estimate can be obtained using EM algorithm. A change-point method of choosing optimal degrees of the proposed Bernstein polynomial model is presented. Under some conditions the optimal rate of convergence in the mean $\chi^{2}$-divergence of new density estimator is shown to be nearly parametric. The method is illustrated by an application to a real data set. Finite sample performance of the proposed method is also investigated by simulation study and is shown to be much better than the kernel density estimate but close to the parametric ones.
\end{abstract}

\section{KEYWORDS}

Approximate Bernstein polynomial model; Beta mixture; Maximum likelihood; Multivariate density estimation; Nonparametric model.

CONTACT Zhong Guan. Email: zguan@iusb.edu 


\section{Introduction}

In nonparametric statistics, density estimation is a difficult job. Multivariate density estimation is even more difficult. A complete account of the multivariate density estimation can be found in the book by Scott [1. The most commonly used method of multivariate density estimation is kernel estimation. Some modifications on kernel density estimation can be found in [2 4]. However, the kernel method is not a maximum likelihood method. It is just a technique to smooth the discrete density corresponding to the empirical distribution by choosing appropriate bandwidth. The kernel density is actually an unbiased estimate of the convolution of the target density and the scaled kernel. Moreover, the boundary effect of kernel estimation and the difficulty in selecting the bandwidth still prevent the improvement upon the accuracy of estimation.

All nonparametric estimates of infinite-dimensional parameters such as continuous distribution and density functions are based on models with finite-dimensional parameter. For example, the commonly used empirical distribution is based on multinomial distribution model. Empirical likelihood method uses the same model. This model can also be viewed as a step-function approximation of the unknown population distribution function or histogram approximation of the underlying density function. However it is not a smooth approximation.

If a nonparametric model for density means any nonnegative function $f$ such that $\int f(x) d x=1$, then for any $x$ with $f(x)>0$, the information for $f(x)$ is zero [see [5]. It was also showed by [6] that no such nonparametric model even with some smoothness assumptions for which this information is positive. Therefore such 'nonparametric model' is not useful. Box [7] noted 'all models are wrong, but some are useful'. If this is agreed then such infinite dimensional 'nonparametric models' are not even models 
because they are not wrong and specify almost nothing. Therefore properly reducing the infinite dimensional parameter to a finite dimensional one is necessary. It is well known that in most cases the maximum likelihood method gives the most efficient estimate. A working finite dimensional nonparametric density model is also necessary to apply the maximum likelihood method. Just like we clearly know when an estimator is parametric, with an approximate working finite dimensional nonparametric density model, one can easily answer the question: 'when is an estimator nonparametric?' [see $\S \S 2.4 .2,6.1 .3$, and 6.4 of 1

Inspired by the Bernstein [8] polynomial approximation [see 10]11, also.], Guan [12] proposed the approximate Bernstein polynomial model for nonparametric density estimation. The unknown parameters contained in this model are the coefficients and the degree of the polynomial which is also the number of unknown coefficients. The number of parameters could increase as sample size increases. This is one of important features that characterize a nonparametric estimator [see $\S 2.4 .2$ of 1 . The readers are refereed to [12]13] for more references therein on applications of the Bernstein polynomial in estimations of density and other smooth infinite dimensional parameters. Unlike other nonparametric density estimation such as the kernel density and other applications of the Bernstein polynomial in density estimations as in [14] and [15], for instance, Guan [12]'s method is a maximum likelihood method using Bernstein polynomial as an approximate model with the degree of the polynomial together with the coefficients as a finite dimensional parameter. It has been shown that the Bernstein density estimation can achieve an almost parametric optimal rate of convergence. Simulation study showed that the small sample performance of the Bernstein polynomial density estimate is close to that of parametric one but much better than the kernel density estimate. 
Multivariate density estimation is crucial in many applications of statistics. For example the Nadaraya-Watson estimator of a nonparametric regression function requires the nonparametric multivariate density estimate. The commonly used kernel density estimator cannot take the advantage of the boundedness of the support of a density to be estimated and therefore its rate of convergence is bounded by the optimal minimax rate [16]. On the other hand, however, for a density on an infinite support it is not possible to obtain reasonable estimates of the density values outside the data range without specification of the tail behaviors. The multivariate generalization of [12]'s method is desired and is anticipated to provide better nonparametric multivariate density estimate than the existing methods such as the kernel density estimation.

The paper is organized as follows. We shall give the maximum approximate Bernstein likelihood method in Section 2 and some asymptotic results in Section 3 The proposed methods are compared with some existing competitors through Monte Carlo experiments in Section 4 and are illustrated by a real dataset in Section 5 . The performance of the change-point method for choosing optimal degrees is also studied in Section 4. Further remarks and comments are given in Section6. The proofs of the theoretical results are relegated to the Appendix.

\section{Methodology}

\subsection{Notations}

In this section we first give some notations and definitions that will be used in the following sections. Throughout the paper, we use bold face letters to denote vectors. For example, $\boldsymbol{x}=\left(x_{1}, \ldots, x_{d}\right)^{\mathrm{T}}$ is a $d$-dimensional vector. Inequality $\boldsymbol{x} \leq \boldsymbol{y}$ is understood componentwise, i.e., $x_{j} \leq y_{j}$ for all $j=1, \ldots, d$. The strict inequality 
$\boldsymbol{x}<\boldsymbol{y}$ means $\boldsymbol{x} \leq \boldsymbol{y}$ but $\boldsymbol{x} \neq \boldsymbol{y}$. We denote the taxicab norm by $|\boldsymbol{x}|=\sum_{i=1}^{d}\left|x_{i}\right|$. Let $C^{(k)}[0,1]^{d}$ denote the class of functions $f$ on $[0,1]^{d}$ that have continuous partial derivatives $f^{(\boldsymbol{l})}(\boldsymbol{t}) \equiv \partial^{\langle\boldsymbol{l}\rangle} f(\boldsymbol{t}) / \partial t_{1}^{l_{1}} \cdots \partial t_{d}^{l_{d}}$, where $0 \leq\langle\boldsymbol{l}\rangle \equiv l_{1}+\cdots+l_{d} \leq k$.

The density of beta distribution with shape parameters $(i+1, m-i+1)$ is

$$
\beta_{m i}(t)=(m+1)\left(\begin{array}{c}
m \\
i
\end{array}\right) t^{i}(1-t)^{m-i}, \quad i=0, \ldots, m ; 0 \leq t \leq 1
$$

Then the generalized multivariate Bernstein polynomial, the multivariate polynomial with positive coefficients, can be defined as

$$
P_{\boldsymbol{m}}(\boldsymbol{t})=\sum_{\boldsymbol{i}=0}^{\boldsymbol{m}} a(\boldsymbol{i}) \cdot \beta_{\boldsymbol{m i}}(\boldsymbol{t}), \quad a(\boldsymbol{i}) \geq 0
$$

where $\boldsymbol{t}=\left(t_{1}, \ldots, t_{d}\right), \boldsymbol{m}=\left(m_{1}, \ldots, m_{d}\right), \boldsymbol{i}=\left(i_{1}, \ldots, i_{d}\right), \sum_{\boldsymbol{i}=0}^{\boldsymbol{m}}=\sum_{i_{1}=0}^{m_{1}} \cdots \sum_{i_{d}=0}^{m_{d}}$ and $\beta_{\boldsymbol{m i}}(\boldsymbol{t})=\prod_{j=1}^{d} \beta_{m_{j} i_{j}}\left(t_{j}\right)$. The maximum number of nonzero coefficients is $K=$ $\prod_{j=1}^{d}\left(m_{j}+1\right)$.

\subsection{Maximum Approximate Bernstein Likelihood Estimation}

Let $\boldsymbol{X}=\left(X_{1}, \ldots, X_{d}\right)^{\mathrm{T}}$ be a $d$-dimensional random vector having a continuous joint distribution $F$ and a density $f$ with support inside the hypercube $[0,1]^{d}$. The part (iii) of Lemma A.1 in the Appendix implies that we can model the multivariate density $f$ approximately by

$$
f_{\boldsymbol{m}}(\boldsymbol{t} ; \boldsymbol{p})=\sum_{\boldsymbol{i}=0}^{\boldsymbol{m}} p(\boldsymbol{i}) \cdot \beta_{\boldsymbol{m i}}(\boldsymbol{t}),
$$

where $\boldsymbol{p}=\boldsymbol{p}_{\boldsymbol{m}}=\{p(\boldsymbol{i}): \mathbf{0} \leq \boldsymbol{i} \leq \boldsymbol{m}\} \in \mathbb{S}_{\boldsymbol{m}} \equiv\{p(\boldsymbol{i}): \mathbf{0} \leq \boldsymbol{i} \leq \boldsymbol{m}, p(\boldsymbol{i}) \geq$ $\left.0, \quad \sum_{\boldsymbol{i}=0}^{\boldsymbol{m}} p(\boldsymbol{i})=1\right\}$, the $(K-1)$-simplex. Then $f_{\boldsymbol{m}}$ is a mixture density of $K$ multiple beta distributions. Moreover, the marginal densities are also mixtures of (multiple) 
beta densities. The joint cdf $F$ can be approximated by

$$
F_{\boldsymbol{m}}(\boldsymbol{t} ; \boldsymbol{p})=\sum_{\boldsymbol{i}=0}^{\boldsymbol{m}} p\left(i_{1}, \ldots, i_{d}\right) \cdot B_{\boldsymbol{m i}}(\boldsymbol{t}),
$$

where $B_{\boldsymbol{m i}}(\boldsymbol{t})=\prod_{j=1}^{d} B_{m_{j} i_{j}}\left(t_{j}\right)$ and $B_{m i}(t)$ is the cumulative distribution function of $\operatorname{beta}(i+1, m-i+1), i=0, \ldots, m$.

Let $\boldsymbol{x}_{i}=\left(x_{1 i}, \ldots, x_{d i}\right)^{\mathrm{T}}, i=1, \ldots, n$, be a sample of size $n$ from $F$. We assume that $\boldsymbol{p}_{\boldsymbol{m}}$ is arranged in the lexicographical order of $\boldsymbol{i}=\left(i_{1}, \ldots, i_{d}\right)$ so that $\boldsymbol{p}_{\boldsymbol{m}}$ can be treated as a $K$-dimensional vector. We can define the approximate Bernstein log-likelihood

$$
\ell\left(\boldsymbol{p}_{\boldsymbol{m}}\right)=\sum_{k=1}^{n} \log f_{\boldsymbol{m}}\left(\boldsymbol{x}_{k} ; \boldsymbol{p}\right)=\sum_{k=1}^{n} \log \left\{\sum_{\boldsymbol{i}=0}^{\boldsymbol{m}} p(\boldsymbol{i}) \beta_{\boldsymbol{m} \boldsymbol{i}}\left(\boldsymbol{x}_{k}\right)\right\}
$$

It is easy to see that if $n \geq K-1$ then $\ell\left(\boldsymbol{p}_{\boldsymbol{m}}\right)$, as a function of $\boldsymbol{p}_{\boldsymbol{m}}$, is strictly concave with probability one. The maximizer $\hat{\boldsymbol{p}}_{\boldsymbol{m}}$ of $\ell\left(\boldsymbol{p}_{\boldsymbol{m}}\right)$ subject to constraint $\boldsymbol{p}_{\boldsymbol{m}} \in \mathbb{S}_{\boldsymbol{m}}$ is called the maximum approximate Bernstein likelihood estimate (MABLE) of $\boldsymbol{p}_{\boldsymbol{m}}$. We can estimate the underlying density and distribution functions, respectively, by the maximum approximate Bernstein likelihood estimators (MABLEs) $\hat{f}_{\mathrm{B}}(\boldsymbol{t})=f_{\boldsymbol{m}}(\boldsymbol{t} ; \hat{\boldsymbol{p}})=$ $\sum_{\boldsymbol{i}=\mathbf{0}}^{\boldsymbol{m}} \hat{p}(\boldsymbol{i}) \beta_{\boldsymbol{m i}}(\boldsymbol{t})$ and $\hat{F}_{\mathrm{B}}(\boldsymbol{t})=F_{\boldsymbol{m}}(\boldsymbol{t} ; \hat{\boldsymbol{p}})=\sum_{\boldsymbol{i}=\mathbf{0}}^{\boldsymbol{m}} \hat{p}(\boldsymbol{i}) B_{\boldsymbol{m i}}(\boldsymbol{t})$.

\subsection{Optimal Degrees}

Starting with an initial value $\boldsymbol{p}_{\boldsymbol{m}}^{(0)}$, one can use the following iteration to find the maximum likelihood estimate of $\boldsymbol{p}_{\boldsymbol{m}}$ for any given $\boldsymbol{m}$ :

$$
\begin{gathered}
p^{(s+1)}\left(l_{1}, \ldots, l_{d}\right)=\frac{1}{n} \sum_{j=1}^{n} \frac{p^{(s)}\left(l_{1}, \ldots, l_{d}\right) \prod_{v=1}^{d} \beta_{m_{v} l_{v}}\left(x_{v j}\right)}{\sum_{i=0}^{m} p^{(s)}\left(i_{1}, \ldots, i_{d}\right) \prod_{v=1}^{d} \beta_{m_{v} i_{v}}\left(x_{v j}\right)}, \\
0 \leq l_{v} \leq m_{v} ; \quad 1 \leq v \leq d ; s=0,1, \ldots
\end{gathered}
$$

It follows from Theorem 4.2 of [17] that for each $\boldsymbol{m}$, as $s \rightarrow \infty, \boldsymbol{p}_{\boldsymbol{m}}^{(s)}$ converges to $\hat{\boldsymbol{p}}_{\boldsymbol{m}}$. 
Because the marginal density of $X_{j}$ can be approximated by a mixture of the $\left(m_{j}+1\right)$ beta densities, $\operatorname{beta}\left(i+1, m_{j}-i+1\right), i=0, \ldots, m_{j}$, [12] gives a lower bound for $m_{j}$ which is $m_{b j}=\max \left\{1,\left\lceil\mu_{j}\left(1-\mu_{j}\right) / \sigma_{j}^{2}-3\right\rceil\right\}$, where $\mu_{j}=\mathrm{E}\left(X_{j}\right)$ and $\sigma_{j}^{2}=\operatorname{var}\left(X_{j}\right)$. One can estimate $\mu_{j}$ and $\sigma_{j}^{2}$, respectively, by $\hat{\mu}_{j}=\bar{x}_{j} .=\frac{1}{n} \sum_{i=1}^{n} x_{j i}, \hat{\sigma}_{j}^{2}=s_{j}^{2}=$ $\frac{1}{n-1} \sum_{i=1}^{n}\left(x_{j i}-\bar{x}_{j .}\right)^{2}$. We can select the optimal $m_{j}$ using the change-point method of [12] for each $j$. Let $\mathfrak{M}_{j}=\left\{m_{j i}=m_{j 0}+i, i=0,1, \ldots, k_{j}\right\}$. We fit the marginal data $x_{j 1}, \ldots, x_{j n}$, with the Bernstein model of degree $m_{j i} \in \mathfrak{M}_{j}$ to obtain the profile $\log$-likelihood $\ell_{j i}=\ell_{j}\left(m_{j i}\right)$. Let $y_{j i}=\ell_{j i}-\ell_{j, i-1}, i=1, \ldots, k_{j}$. We heuristically assume that $y_{j 1}, \ldots, y_{j n}$ are exponentials with a change point $\tau_{j}$ and that $m_{j \tau_{j}}$ is the optimal degree. We use the change-point detection method [see Section 1.4 of 18 for exponential model to find a change-point estimate $\hat{\tau}_{j}=\arg \max _{1 \leq \tau \leq k_{j}}\left\{R_{j}(\tau)\right\}$, where the likelihood ratio of $\tau$ is

$$
R_{j}(\tau)=-\tau \log \left(\frac{\ell_{j \tau}-\ell_{j 0}}{\tau}\right)-\left(k_{j}-\tau\right) \log \left(\frac{\ell_{j k}-\ell_{j \tau}}{k_{j}-\tau}\right)+k_{j} \log \left(\frac{\ell_{j k_{j}}-\ell_{j 0}}{k_{j}}\right),
$$

for $\tau=1, \ldots, k_{j}$.Then we estimate the optimal $m_{j}$ by $\hat{m}_{j}=m_{j \hat{\tau}_{j}}$. In case $R_{j}(\tau)$ has multiple maximizers, we choose the smallest one as $\hat{\tau}_{j}$.

\subsection{Multivariate Distribution and Density Functions Estimation}

Let $\boldsymbol{Y}=\left(Y_{1}, \ldots, Y_{d}\right)^{\mathrm{T}}$ be a $d$-dimensional random vector having a continuous joint distribution $G$ and a density $g$ with support $\left[a_{1}, b_{1}\right] \times \cdots \times\left[a_{d}, b_{d}\right]$. We transform $\boldsymbol{Y}$ to $\boldsymbol{X}=\left(X_{1}, \ldots, X_{d}\right)^{\mathrm{T}}$, where $X_{i}=\left(Y_{i}-a_{i}\right) /\left(b_{i}-a_{i}\right), i=1, \ldots, d$. Then the distribution and density functions of $\boldsymbol{X}$ are, respectively, $F(\boldsymbol{x})=F\left(x_{1}, \ldots, x_{d}\right)=$ $G\{(\boldsymbol{b}-\boldsymbol{a}) \boldsymbol{x}+\boldsymbol{a}\}=G\left\{\left(b_{1}-a_{1}\right) x_{1}+a_{1}, \ldots,\left(b_{d}-a_{d}\right) x_{d}+a_{d}\right\}$ and $f(\boldsymbol{x})=\left(b_{1}-a_{1}\right) \cdots\left(b_{d}-\right.$ $\left.a_{d}\right) g\{(\boldsymbol{b}-\boldsymbol{a}) \boldsymbol{x}+\boldsymbol{a}\}=\left(b_{1}-a_{1}\right) \cdots\left(b_{d}-a_{d}\right) g\left\{\left(b_{1}-a_{1}\right) x_{1}+a_{1}, \ldots,\left(b_{d}-a_{d}\right) x_{d}+a_{d}\right\}$. Let $\boldsymbol{y}_{j}=\left(y_{1 j}, \ldots, y_{d j}\right)^{\mathrm{T}}, j=1, \ldots, n$, be a sample from $G$. We transform the data to 
$\boldsymbol{x}_{j}=\left(x_{1 j}, \ldots, x_{d j}\right)^{\mathrm{T}}$, with $x_{i j}=\left(y_{i j}-a_{i}\right) /\left(b_{i}-a_{i}\right), j=1, \ldots, n, i=1, \ldots, d$. Since $f$ is a continuous $d$-variate density on the hypercube $[0,1]^{d}$, we can fit the transformed data $\boldsymbol{x}_{j}, j=1, \ldots, n$, by the Bernstein polynomial model to get the maximum likelihood estimate $\hat{\boldsymbol{p}}$. Then we can estimate $g$ and $G$ respectively by

$$
\begin{aligned}
\hat{g}_{\mathrm{B}}(\boldsymbol{y}) & =\frac{1}{\prod_{i=1}^{d}\left(b_{i}-a_{i}\right)} \sum_{i=0}^{\boldsymbol{m}} \hat{p}\left(i_{1}, \ldots, i_{d}\right) \cdot \prod_{j=1}^{d} \beta_{m_{j} i_{j}}\left(\frac{y_{j}-a_{j}}{b_{j}-a_{j}}\right), \\
\hat{G}_{\mathrm{B}}(\boldsymbol{y}) & =\sum_{i=0}^{\boldsymbol{m}} \hat{p}\left(i_{1}, \ldots, i_{d}\right) \cdot \prod_{j=1}^{d} B_{m_{j} i_{j}}\left(\frac{y_{j}-a_{j}}{b_{j}-a_{j}}\right) .
\end{aligned}
$$

\section{Asymptotic Results}

In order to prove our asymptotic results we need the following assumption:

(A.1). For each $\boldsymbol{m}$ large enough, there exist a $\boldsymbol{p}_{0} \in \mathbb{S}_{\boldsymbol{m}}$ and $k>0$ such that, uniformly in $\boldsymbol{t} \in(0,1)^{d}$,

$$
\frac{\left|f_{\boldsymbol{m}}\left(\boldsymbol{t} ; \boldsymbol{p}_{0}\right)-f(\boldsymbol{t})\right|}{f(\boldsymbol{t})} \leq C(d, f) m_{0}^{-k / 2}
$$

where $m_{0}=\min _{1 \leq j \leq d} m_{j}$, and $C(d, f)$ depends on $d$ and $f$ but independent of $\boldsymbol{m}$.

A function $f$ is said to be $\gamma$-Hölder continuous with $\gamma \in(0,1]$ if $|f(x)-f(y)| \leq$ $C|x-y|^{\gamma}$ for some constant $C>0$. By Lemma A.1 and Remark 4 we have the following sufficient condition for assumption (A.1) to hold.

Lemma 3.1. Suppose that $f_{0} \in C^{(r)}[0,1]^{d}, r \geq 0, f_{0}(\boldsymbol{t}) \geq \delta_{0}>0$, and when $\langle\boldsymbol{l}\rangle=r$ all $f_{0}^{(\boldsymbol{l})}$ are $\alpha$-Hölder continuous, $\alpha \in(0,1]$. If $f(\boldsymbol{t})=f_{0}(\boldsymbol{t}) \prod_{i=1}^{d} t_{i}^{a_{i}}\left(1-t_{i}\right)^{b_{i}}$, where $a_{i}$ 's and $b_{i}$ 's are nonnegative integers, then assumption (A.1) is true with $k=r+\alpha$.

Intuitively, assumption (A.1) suggests that most of sample data can be viewed as if they were from $f_{\boldsymbol{m}}\left(\boldsymbol{t} ; \boldsymbol{p}_{0}\right)$ [13]. So $\ell(\boldsymbol{p})$ is the likelihood of $\boldsymbol{x}_{1}, \ldots, \boldsymbol{x}_{n}$ which can 
be viewed as a slightly contaminated sample from $f_{\boldsymbol{m}}\left(\boldsymbol{t} ; \boldsymbol{p}_{0}\right)$. Hence $f_{\boldsymbol{m}}(\boldsymbol{t} ; \hat{\boldsymbol{p}})$ approximately targets at $f_{\boldsymbol{m}}\left(\boldsymbol{t} ; \boldsymbol{p}_{0}\right)$ which is an approximation of $f$ satisfying assumption (A.1), For a given $\boldsymbol{p} \in \mathbb{S}_{\boldsymbol{m}}$, we define the $\chi^{2}$-divergence $\left(\chi^{2}\right.$-distance)

$$
D^{2}(\boldsymbol{p})=\int_{[0,1]^{d}} \frac{\left\{f_{m}(\boldsymbol{t} ; \boldsymbol{p})-f(\boldsymbol{t})\right\}^{2}}{f(\boldsymbol{t})} d \boldsymbol{t} \equiv \int_{[0,1]^{d}}\left[\frac{f_{m}(\boldsymbol{t} ; \boldsymbol{p})}{f(\boldsymbol{t})}-1\right]^{2} f(\boldsymbol{t}) d \boldsymbol{t}
$$

For density estimation we have the following result.

Theorem 3.2. Under assumption (A.1) for some positive integer $k$, and $m_{0}=$ $\mathcal{O}\left(n^{1 / k}\right)$. As $n \rightarrow \infty$, with probability one the maximum value of $\ell(\boldsymbol{p})$ is attained by some $\hat{\boldsymbol{p}}$ in the interior of $\mathbb{B}_{\boldsymbol{m}}\left(r_{n}\right)=\left\{\boldsymbol{p} \in \mathbb{S}_{\boldsymbol{m}}: D^{2}(\boldsymbol{p}) \leq r_{n}^{2}\right\}$, where $r_{n}^{2}=\log n / n$. Consequently we have

$$
\mathrm{E} \int_{[0,1]^{d}} \frac{\left\{f_{\boldsymbol{m}}(\boldsymbol{t} ; \hat{\boldsymbol{p}})-f(\boldsymbol{t})\right\}^{2}}{f(\boldsymbol{t})} d \boldsymbol{t} \leq \frac{\log n}{n} .
$$

Because $f$ is bounded there is a positive constant $C$ such that

$$
\operatorname{MISE}\left(\hat{f}_{\mathrm{B}}\right)=\mathrm{E} \int_{[0,1]^{d}}\left\{f_{\boldsymbol{m}}(\boldsymbol{t} ; \hat{\boldsymbol{p}})-f(\boldsymbol{t})\right\}^{2} d \boldsymbol{t} \leq C \frac{\log n}{n} .
$$

Remark 1. The result (8) is a stronger result than (9) because $f$ can be arbitrarily small. The rate $(9)$ is an almost parametric rate of convergence for MISE. This rate can be attained by kernel type estimators for analytic densities [19. It is an interesting to investigate the properties of the proposed method for analytic density functions. Guan [12] showed a similar result when $d=1$ under another set of conditions. The best rate is $\mathcal{O}\left(n^{-1}\right)$ that can be attained by the parametric density estimate under some regularity conditions.

Remark 2. The Remark 1 of [20] mentioned a minimax rate of $\mathcal{O}\left(n^{-2 k /(2 k+1)}\right)$ for a larger Hölder class of order $k$ of univariate density functions even with restriction to $[0,1]$ [see 21, also]. This does not contradict our result because we consider a smaller 
class of density functions which satisfy assumption (A.1) while a Hölder class density does not necessrily fulfill this assumption. For example, $f(x)=[x(1-x)]^{r+\alpha} / B(a, b)$, the density of beta distribution with shapes $a=b=r+1+\alpha$, where $r$ is nonnegative integer and $0<\alpha<1$. This is a member of Hölder class of order $k=r+\alpha$ but does not satisfy assumption (A.1).

\section{Simulation Study}

Consider the two-dimensional random vector $\left(X_{1}, X_{2}\right)^{\mathrm{T}}$ with pdf $f\left(x_{1}, x_{2}\right)$ and support $[0,1]^{2}$. We generate random samples of size $n$ from some distributions. Simulation results on the estimated optimal model degrees $\hat{\boldsymbol{m}}$ and mean integrated squared errors $(\times 100)$ of the density estimates based 1000 Monte Carlo runs are given in Table 1 In this table, $\hat{f}_{\mathrm{B}}$ represents the maximum approximate Bernstein likelihood density estimate; $\hat{f}_{\mathrm{K}}$ the kernel density computed by $\mathrm{R}$ package "ks". Samples of size $n$ are generated from the following distributions.

(i) Beta: joint beta distribution with pdf $f\left(x_{1}, x_{2}\right)=\operatorname{beta}\left(x_{1} ; 7,7\right) \operatorname{beta}\left(x_{2} ; 5,5\right)$.

(ii) Normal: bivariate normal with mean $\boldsymbol{\mu}=(0.5,0.5)^{\mathrm{T}}$ and covariance matrix $\boldsymbol{\Sigma}=$ $0.125^{2}\left(\begin{array}{ll}1 & 0.1 \\ 0.1 & 1\end{array}\right)$

(iii) MN: bivariate normal mixture $w_{1} N\left(\boldsymbol{\mu}_{1}, \boldsymbol{\Sigma}_{1}\right)+w_{2} N\left(\boldsymbol{\mu}_{2}, \boldsymbol{\Sigma}_{2}\right)$ with parameters $w=\left(w_{1}, w_{2}\right)=(0.3,0.7), \boldsymbol{\mu}_{1}=(0.3,0.3)^{\mathrm{T}}, \boldsymbol{\mu}_{2}=(0.7,0.7)^{\mathrm{T}}$,

$$
\boldsymbol{\Sigma}_{1}=\left(\begin{array}{lr}
0.065^{2} & 0 \\
0 & 0.065^{2}
\end{array}\right), \boldsymbol{\Sigma}_{2}=\left(\begin{array}{lr}
0.065^{2} & 0 \\
0 & 0.065^{2}
\end{array}\right)
$$

(iv) $\mathrm{P}(8,8)$ : power distribution with pdf $f\left(x_{1}, x_{2}\right)=4.5\left(x_{1}^{8}+x_{2}^{8}\right), 0 \leq x_{1}, x_{2} \leq 1$.

From Table 1 we observe the following. The change-point method for choosing optimal degrees seems to give consistent estimate of $\boldsymbol{m}$ for beta distribution. For non- 
Table 1. Simulation using optimal degree $m$. Simulation results on estimated optimal model degree $\hat{\boldsymbol{m}}$ and mean integrated square errors $(\times 100)$ of density estimates based 1000 Monte Carlo runs. $\hat{f}_{\mathrm{B}}$ : maximum Bernstein likelihood density estimate; $\hat{f}_{\mathrm{K}}$ : kernel density.

\begin{tabular}{|c|c|c|c|c|}
\hline & Beta & Normal & $\mathrm{MN}$ & $\mathrm{P}(8,8)$ \\
\hline \multicolumn{5}{|c|}{$n=20$} \\
\hline $\mathrm{E}(\hat{\boldsymbol{m}})$ & $(10.90,8.72)$ & $(11.50,11.55)$ & $(43.83,44.17)$ & $(6.25,6.26)$ \\
\hline $\mathrm{SD}(\hat{\boldsymbol{m}})$ & $(3.04,3.08)$ & $(3.10,2.99)$ & $(12.29,12.53)$ & $(2.51,2.54)$ \\
\hline $\operatorname{MISE}\left(\hat{f}_{\mathrm{B}}\right)$ & 30.438 & 44.814 & 151.846 & 153.602 \\
\hline $\operatorname{MISE}\left(\hat{f}_{\mathrm{K}}\right)$ & 63.957 & 86.723 & 217.632 & 206.481 \\
\hline \multicolumn{5}{|c|}{$n=50$} \\
\hline $\mathrm{E}(\hat{\boldsymbol{m}})$ & $(11.42,8.66)$ & $(12.29,12.25)$ & $(46.05,44.80)$ & $(5.86,5.92)$ \\
\hline $\mathrm{SD}(\hat{\boldsymbol{m}})$ & $(2.41,2.26)$ & $(2.53,2.55)$ & $(10.54,9.95)$ & $(1.99,2.09)$ \\
\hline $\operatorname{MISE}\left(\hat{f}_{\mathrm{B}}\right)$ & 13.812 & 23.427 & 75.429 & 79.496 \\
\hline $\operatorname{MISE}\left(\hat{f}_{\mathrm{K}}\right)$ & 32.680 & 43.904 & 127.248 & 177.962 \\
\hline \multicolumn{5}{|c|}{$n=100$} \\
\hline $\mathrm{E}(\hat{\boldsymbol{m}})$ & $(11.79,8.58)$ & $(12.87,12.96)$ & $(46.68,46.27)$ & $(5.365 .31)$ \\
\hline $\mathrm{SD}(\hat{\boldsymbol{m}})$ & $(2.04,1.79)$ & $(2.20,2.23)$ & $(8.13,8.18)$ & $(1.24,1.25)$ \\
\hline $\operatorname{MISE}\left(\hat{f}_{\mathrm{B}}\right)$ & 7.403 & 14.037 & 43.340 & 49.420 \\
\hline $\operatorname{MISE}\left(\hat{f}_{\mathrm{K}}\right)$ & 20.238 & 27.691 & 82.454 & 158.145 \\
\hline \multicolumn{5}{|c|}{$n=200$} \\
\hline $\mathrm{E}(\hat{\boldsymbol{m}})$ & $(11.99,8.46)$ & $(13.22,13.13)$ & $(48.24,48.08)$ & $(5.95,5.92)$ \\
\hline $\mathrm{SD}(\hat{\boldsymbol{m}})$ & $(1.56,1.48)$ & $(1.87,1.77)$ & $(6.85,6.63)$ & $(1.33,1.33)$ \\
\hline $\operatorname{MISE}\left(\hat{f}_{\mathrm{B}}\right)$ & 3.733 & 8.884 & 24.407 & 33.672 \\
\hline $\operatorname{MISE}\left(\hat{f}_{\mathrm{K}}\right)$ & 12.943 & 17.453 & 53.237 & 141.669 \\
\hline
\end{tabular}


polynomial density distributions the optimal degrees seems increase slowly as sample size $n$ with decreasing standard deviation. The proposed density estimate $\hat{f}_{\mathrm{B}}$ could be 3 times and at least 1.3 times more efficient than the kernel density $\hat{f}_{\mathrm{K}}$. The relative efficiency of $\hat{f}_{\mathrm{B}}$ to $\hat{f}_{\mathrm{K}}$ seems increases as $n$.

\section{Real Data Application}

The joint density of duration $Y_{1}$ (in minutes) of eruptions and the waiting time $Y_{2}$ (in minutes) of the Old Faithful is bimodal. Based on the data set containing $n=272$ observations which are contained in [22] and also in [23. Density estimation based on these data are also discussed by [24] and [23]. Petrone [25] provides a comparison with the Baysian Bernstein density estimate.

We truncate the data by rectangle $\left[a_{1}, b_{1}\right] \times\left[a_{2}, b_{2}\right]=[0,7] \times[0,120]$ and transform the data to $X_{i}=\left(Y_{i}-a_{i}\right) /\left(b_{i}-a_{i}\right), i=1,2$. Using the change-point method of [12 as described in Section 2.3 we obtained optimal degrees $\hat{m}_{1}=95$ and $\hat{m}_{2}=88$ by fitting the duration and waiting time data separately with the Bernstein polynomial model. The maximum approximate Bernstein likelihood estimate $\hat{g}_{\mathrm{B}}$ of the truncated density based on the data $Y_{i}^{\prime}$ 's with $\hat{\boldsymbol{m}}=(95,88)$ is transformed to give the maximum approximate Bernstein likelihood estimate $\hat{f}_{\mathrm{B}}$ of $f$ : $\hat{f}_{\mathrm{B}}(\boldsymbol{x})=\hat{g}_{\mathrm{B}}\left\{\left(x_{1}-a_{1}\right) /\left(b_{1}-a_{1}\right),\left(x_{2}-a_{2}\right) /\left(b_{2}-a_{2}\right)\right\} /\left[\left(b_{1}-a_{1}\right)\left(b_{2}-a_{2}\right)\right]$. Figure 1 shows the density estimates, the proposed method of this paper $\hat{f}_{\mathrm{B}}$, the kernel density $\hat{f}_{\mathrm{K}}$ using $\mathrm{R}$ package ks based on mixture data, and the parametric estimate $\hat{f}_{\mathrm{P}}$ using $\mathrm{R}$ package mixtools, the mixture normal model, and the histogram $\hat{f}_{\mathrm{H}}$ of the data. Figure 2 compares the four estimates by contours. From these graphs we see that the proposed estimate is a little bit more smooth and looks more like mixture normal as many authors have assumed. 

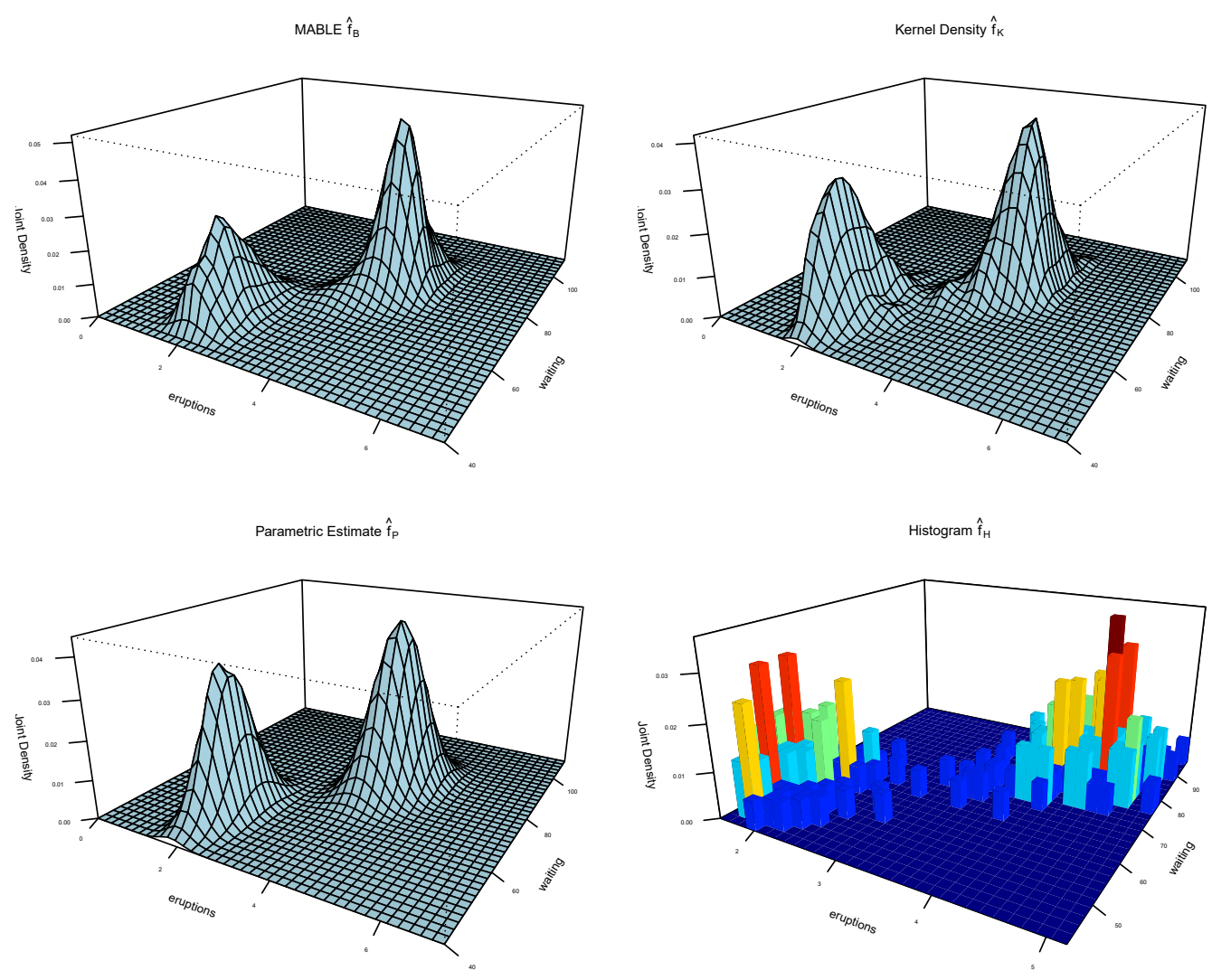

Figure 1. $\hat{f}_{\mathrm{B}}$ : the Bernstein density estimate; $\hat{f}_{\mathrm{K}}$ : the kernel density estimate; $\hat{f}_{\mathrm{P}}$ : the mixed normal density estimate; $\hat{f}_{\mathrm{H}}$ : the histogram estimate. 

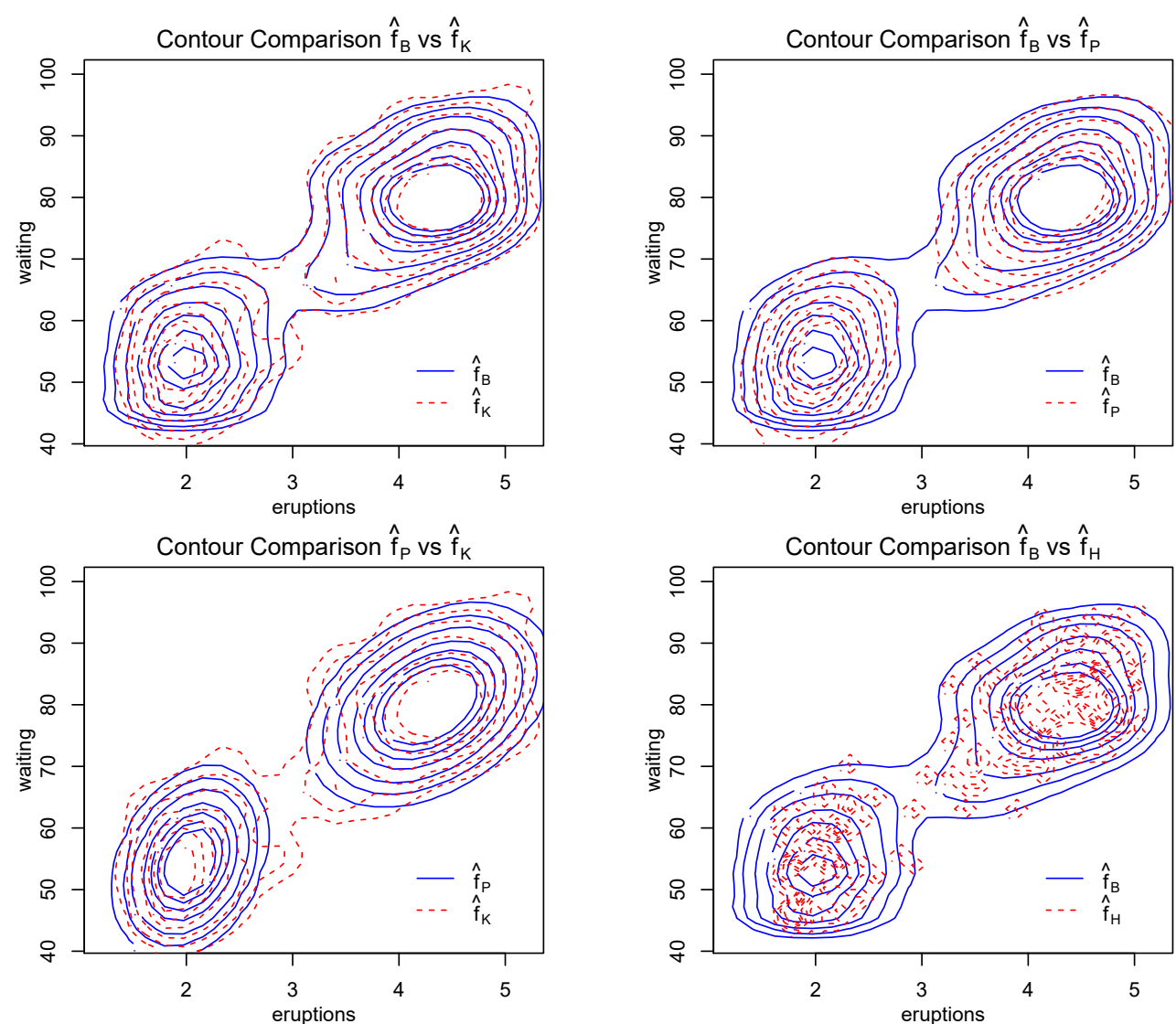

Figure 2. Pairwise contour comparison of the density estimates. $\hat{f}_{\mathrm{B}}$ : the Bernstein density estimate; $\hat{f}_{\mathrm{K}}$ : the kernel density estimate; $\hat{f}_{\mathrm{P}}$ : the mixed normal density estimate; $\hat{f}_{\mathrm{H}}$ : the histogram estimate. 


\section{Concluding Remark}

Due to the many parameters to be estimated, the only drawback of the proposed method is the slow convergence of EM iteration. The computation cost seems unavoidable to achieve the much better efficiency. It is a challenge to find better algorithm to speed up the computation. Although assumption (A.1) is not easy to check, the sufficient condition given in Lemma 3.1 with all $a_{i}$ 's and $b_{i}$ 's equal to zero is fulfilled by non-vanishing densities. A nonparametric density estimator should be obtained by fitting a working nonparametric model for density. A useful and working nonparametric model should be an approximate model which contains unknown but finite number of parameters. The method described in this is implemented in $\mathrm{R}$ language [26] and will be added as a component to $\mathrm{R}$ package mable, maximum approximate Bernstein likelihood estimation [27, which is available on CRAN.

\section{Funding}

Tao Wang is supported by the Natural Science Foundation of Heilongjiang Province, China(Granted No. A2017006).

\section{References}

[1] Scott DW. Multivariate density estimation: theory, practice, and visualization. John Wiley \& Sons; 2015.

[2] Romano J. On weak convergence and optimality of kernel density estimates of the mode. The Annals of Statistics. 1988;16(2):629-647.

[3] Tran LT. On multivariate variable-kernel density estimates for time series. The Canadian Journal of Statistics. 1988;19(4):371-387. 
[4] Vieu P. A note on density mode estimation. Statistics \& Probability Letters. 1996; 26(4):297-307.

[5] Bickel PJ, Klaassen CAJ, Ritov Y, et al. Efficient and adaptive estimation for semiparametric models. New York: Springer-Verlag; 1998. Reprint of the 1993 original.

[6] Ibragimov I, Khasminskii R. Estimation of distribution density belonging to a class of entire functions. Theory of Probability \& Its Applications. 1983;27(3):551-562.

[7] Box GEP. Science and statistics. Journal of the American Statistical Association. 1976; 71(356):791-799.

[8] Bernstein SN. Démonstration du théorème de Weierstrass fondée sur le calcul des probabilitiés. Communications of the Kharkov Mathematical Society. 1912;13:1-2.

[9] Bernstein SN. Complétement à l'article de E. Voronowskaja. C R Acad Sci URSS. 1932; $: 86-92$.

[10] Lorentz GG. The degree of approximation by polynomials with positive coefficients. Mathematische Annalen. 1963;151:239-251.

[11] Lorentz GG. Bernstein polynomials. 2nd ed. New York: Chelsea Publishing Co.; 1986.

[12] Guan Z. Efficient and robust density estimation using Bernstein type polynomials. Journal of Nonparametric Statistics. 2016;28(2):250-271.

[13] Guan Z. Bernstein polynomial model for grouped continuous data. Journal of Nonparametric Statistics. 2017;29(4):831-848.

[14] Vitale RA. Bernstein polynomial approach to density function estimation. In: Statistical inference and related topics (proc. summer res. inst. statist. inference for stochastic processes, indiana univ., bloomington, ind., 1974, vol. 2; dedicated to z. w. birnbaum). New York: Academic Press; 1975. p. 87-99.

[15] Tenbusch A. Two-dimensional Bernstein polynomial density estimators. Metrika. 1994; $41(3-4): 233-253$.

[16] Stone CJ. Optimal rates of convergence for nonparametric estimators. The Annals of Statistics. 1980 11;8(6):1348-1360. 
[17] Redner RA, Walker HF. Mixture densities, maximum likelihood and the EM algorithm. SIAM Review. 1984;26(2):195-239.

[18] Csörgő M, Horváth L. Limit theorems in change-point analysis. John Wiley \& Sons, Ltd., Chichester; 1997. Wiley Series in Probability and Statistics; with a foreword by David Kendall.

[19] Stepanova N. On estimation of analytic density functions in $L_{p}$. Mathematical Methods of Statistics. 2013;22(2):114-136.

[20] Juditsky A, Lambert-Lacroix S. On minimax density estimation on R. Bernoulli. 2004 04; 10(2):187-220.

[21] Ibragimov IA, Has' minskiĭ RZ. Statistical estimation. (Applications of Mathematics; Vol. 16). Springer-Verlag, New York-Berlin; 1981. Asymptotic theory, Translated from the Russian by Samuel Kotz.

[22] Härdle W. Smoothing techniques with implementation in S. New York: Springer; 1991.

[23] Venables WN, Ripley BD. Modern applied statistics with S-Plus. New York: SpringerVerlag Inc; 1994.

[24] Silverman BW. Density estimation for statistics and data analysis. London: Chapman \& Hall; 1986. Monographs on Statistics and Applied Probability.

[25] Petrone S. Bayesian density estimation using Bernstein polynomials. The Canadian Journal of Statistics. 1999;27(1):105-126.

[26] R Core Team. R: A language and environment for statistical computing. Vienna, Austria: R Foundation for Statistical Computing; 2018. Available from: https://www.R-project. org/.

[27] Guan Z. mable: Maximum approximate bernstein likelihood estimation; 2018. R package version 1.0.

[28] Hildebrandt TH, Schoenberg IJ. On linear functional operations and the moment problem for a finite interval in one or several dimensions. Annals of Mathematics. 1933;34(2):317328. 
[29] Butzer PL. Linear combinations of Bernstein polynomials. Canadian Journal of Mathematics. 1953;5:559-567.

[30] Butzer PL. On two-dimensional Bernstein polynomials. Canadian Journal of Mathematics. $1953 ; 5: 107-113$.

[31] Sancetta A, Satchell S. The Bernstein copula and its applications to modeling and approximations of multivariate distributions. Econometric Theory. 2004;20(3):535-562.

[32] Romanovsky V. Note on the moments of a binomial $(p+q)^{n}$ about its mean. Biometrika. $1923 ; 15(3-4): 410-412$.

[33] Qin J, Lawless J. Empirical likelihood and general estimating equations. The Annals of Statistics. 1994;22(1):300-325.

\section{Appendices}

\section{Appendix A. Mathematical Preparation}

We denote the modulus of continuity of function $f$ by $\omega(f, h)=\max _{|\boldsymbol{s}-\boldsymbol{t}|<h} \mid f(\boldsymbol{s})-$ $f(\boldsymbol{t}) \mid, h>0$. Define $\omega_{\boldsymbol{r}}(h)=\omega\left(f^{(\boldsymbol{r})}, h\right), \omega^{(r)}(h)=\max _{\langle\boldsymbol{r}\rangle=r} \omega_{\boldsymbol{r}}(h)$, and $\Delta_{n}=\Delta_{n}(t)=$ $\max \left\{n^{-1}, \delta_{n}(t)\right\}, \delta_{n}=\delta_{n}(t)=\sqrt{t(1-t) / n}$. If $n \leq 4$ then $\Delta_{n}(t)=n^{-1}$ for all $t \in[0,1]$. If $n>4$ then

$$
\Delta_{n}(t)= \begin{cases}\delta_{n}(t), & |t-0.5| \leq 0.5 \sqrt{1-4 / n} \\ n^{-1}, & \text { elsewhere. }\end{cases}
$$

Let $f$ be defined on the hypercube $[0,1]^{d}$. The multivariate Bernstein polynomial approximation [28 30] for $f(\boldsymbol{t})$ is

$$
B_{n}^{f}(\boldsymbol{t})=\sum_{\boldsymbol{i}=0}^{\boldsymbol{m}} f(\boldsymbol{i} / \boldsymbol{m}) \cdot b_{\boldsymbol{m}, \boldsymbol{i}}(\boldsymbol{t}),
$$

where $\frac{\boldsymbol{i}}{\boldsymbol{m}}=\left(\frac{i_{1}}{m_{1}}, \ldots, \frac{i_{d}}{m_{d}}\right), b_{\boldsymbol{m}, \boldsymbol{i}}(\boldsymbol{t})=\prod_{j=1}^{d} b_{m_{j} i_{j}}\left(t_{j}\right)$, and $b_{m i}(t) \equiv\left(\begin{array}{c}m \\ i\end{array}\right) t^{i}(1-t)^{m-i}$, 
$i=0, \ldots, m ; 0 \leq t \leq 1$.

The best degree of approximation by $B_{\boldsymbol{n}}^{f}(\boldsymbol{t})$ is $\mathcal{O}\left(\sum_{j=1}^{d} m_{j}^{-1}\right)$ provided that $f$ has continuous second or even higher partial derivatives. Most of the applications [see 1415, for example] of the Bernstein polynomial so far in statistics are limited to estimating $f\left(\frac{i}{m}\right)$ using empirical distribution. So they are far from optimal if the underlying distribution has a smooth density function. One such example is [31] who used Bernstein polynomial to approximate multivariate distributions in terms of empirical Bernstein copulas.

If $d=1$ and $f$ has a positive lower bound and higher than second order continuous derivatives [10] showed that there exist better choices of nonnegative coefficients than $f\left(i / m_{1}\right)$ which result in the so called polynomial with positive coefficients and degree of approximation better than $\mathcal{O}\left(m_{1}^{-1}\right)$. We shall generalize the result of [10] for univariate polynomial with positive coefficients to multivariate case with a little improvement.

Let $\Lambda_{r}^{(d)}=\Lambda_{r}^{(d)}\left(\delta, M_{0}, M_{r}\right), M_{r}=\left(M_{\boldsymbol{i}}=M_{i_{1}, \ldots, i_{d}}, 2 \leq\langle\boldsymbol{i}\rangle \leq r\right)$, be the class of functions $f(\boldsymbol{t})$ in $C^{(r)}[0,1]^{d}$ with the properties $\delta \leq f(\boldsymbol{t}) \leq M_{0},\left|f^{(\boldsymbol{i})}(\boldsymbol{t})\right| \leq M_{\boldsymbol{i}}$, $\boldsymbol{t} \in[0,1]^{d}$, for some $\delta>0, M_{i} \geq 0,2 \leq\langle\boldsymbol{i}\rangle \leq r$. The following is an enhanced generalization of Theorem 1 of [10] to the multivariate positive polynomial which might be of independent interest.

Lemma A.1. (i) If $f \in C^{(r)}[0,1]^{d}, r=0,1$, then with $C_{r, d}=d+1$

$$
\left|f(\boldsymbol{x})-B_{\boldsymbol{m}}^{f}(\boldsymbol{x})\right| \leq C_{r, d} \omega^{(r)}\left(\max _{1 \leq j \leq d} \delta_{m_{j}}\left(x_{j}\right)\right)\left[\sum_{j=1}^{d} \delta_{m_{j}}\left(x_{j}\right)\right]^{r}, \quad 0 \leq \boldsymbol{x} \leq 1 .
$$

(ii) If $r \geq 2, \delta>0, M_{i} \geq 0$, be given, then there exists a constant $C_{r, d}=$ $C_{r, d}\left(\delta, M_{0}, M_{r}\right)$ such that for each function $f(\boldsymbol{x}) \in \Lambda_{r}^{(d)}\left(\delta, M_{0}, \boldsymbol{M}_{r}\right)$ one can find a sequence $P_{\boldsymbol{m}}(\boldsymbol{x}), \boldsymbol{m} \geq 1$, of polynomials with positive coefficients of degree $\boldsymbol{m}$ satisfy- 
ing

$$
\left|f(\boldsymbol{x})-P_{\boldsymbol{m}}(\boldsymbol{x})\right| \leq C_{r, d} \omega^{(r)}\left(D_{\boldsymbol{m}}(\boldsymbol{x})\right) D_{\boldsymbol{m}}^{r-2}(\boldsymbol{x})\left[\sum_{j=1}^{d} \delta_{m_{j}}\left(x_{j}\right)\right]^{2}, \quad 0 \leq \boldsymbol{x} \leq 1,
$$

where $D_{\boldsymbol{m}}(\boldsymbol{x})=\max _{1 \leq j \leq d} \Delta_{m_{j}}\left(x_{j}\right)$. (iii) If $f \in \Lambda_{r}^{(d)}\left(\delta, M_{0}, \boldsymbol{M}_{r}\right)$ is a probability density function, and $f_{0}^{(l)}$ is $\alpha$-Hölder continuous, $\alpha \in(0,1],\langle\boldsymbol{l}\rangle=r$, then normalizing the coefficients of $B_{\boldsymbol{m}}^{f}(\boldsymbol{x})$ or $P_{\boldsymbol{m}}(\boldsymbol{x})$ we obtain $f_{\boldsymbol{m}}(\boldsymbol{x} ; \boldsymbol{p})=\sum_{\boldsymbol{i}=0}^{\boldsymbol{m}} p(\boldsymbol{i}) \cdot \beta_{\boldsymbol{m i}}(\boldsymbol{x})$ with coefficients $\boldsymbol{p} \in \mathbb{S}_{\boldsymbol{m}}$ which satisfies

$$
\left|f(\boldsymbol{x})-f_{\boldsymbol{m}}(\boldsymbol{x} ; \boldsymbol{p})\right| \leq C_{r, d}^{\prime}\left(\min _{1 \leq i \leq d} m_{i}\right)^{-(r+\alpha) / 2}, \quad 0 \leq \boldsymbol{x} \leq 1,
$$

for some constants $C_{r, d}^{\prime}$.

Remark 3. If $d=1$ and $r \geq 2$, then an improved version of Theorem 1 of [10] is

$$
\left|f(t)-P_{n}(t)\right| \leq C_{r} \delta_{n}^{2}(t) \Delta_{n}^{r-2}(t) \omega_{r}\left(\Delta_{n}(t)\right), \quad 0 \leq t \leq 1, \quad n=1, \ldots
$$

This indicates that the approximation $P_{n}$ for $f$ performs especially good at the boundaries because the errors are zero at $t=0,1$. However, results of [10] do not imply this when $r \geq 2$.

Remark 4. The requirement that $f$ has a positive lower bound $\delta$ can be relaxed to allow $f$ to vanish only along the edges of $[0,1]^{d}$. For example, $f(\boldsymbol{x})=f_{0}(\boldsymbol{x}) \prod_{i=1}^{d} x_{i}^{a_{i}}(1-$ $\left.x_{i}\right)^{b_{i}}$, where $f_{0} \in \Lambda_{r}^{(d)}\left(\delta, M_{0}, M_{r}\right), a_{i}$ 's and $b_{i}$ 's are nonnegative integers. Because $P_{\boldsymbol{m}}(\boldsymbol{x})$ is a polynomial with positive coefficients so is $P_{\boldsymbol{m}}(\boldsymbol{x}) \prod_{i=1}^{d} x_{i}^{a_{i}}\left(1-x_{i}\right)^{b_{i}}$. Thus part (ii) of Lemma A.1 is still true.

Using the notations of [10], we define $T_{n s}(x)=\sum_{k=0}^{n}(k-n x)^{s} p_{n k}(x), s=0,1, \ldots$ It is convenient to denote $\bar{T}_{n s}(x)=n^{-s} T_{n s}(x)$ and $\bar{T}_{n s}^{*}(x)=n^{-s} T_{n s}^{*}(x):=n^{-s} \sum_{k=0}^{n} \mid k-$ $\left.n x\right|^{s} p_{n k}(x), s=0,1, \ldots$ In order to get a non-uniform estimate, we need an improved 
version of Lemma 1 of [10]:

Lemma A.2. For $s \geq 0$ and some constant $A_{s}$

$$
\bar{T}_{n s}^{*}(x) \leq A_{s} \delta_{n}^{2 \wedge s}(x) \Delta_{n}^{0 \vee(s-2)}(x)
$$

where $a \vee b=\max (a, b)$, and $a \wedge b=\min (a, b)$. Particularly $A_{0}=A_{1}=A_{2}=1, A_{3}=2$ and $A_{4}=4$. The equality holds when $s=0,2$.

Remark 5. Lemma 1 of [10] gives $\bar{T}_{n s}^{*}(x) \leq A_{s} \Delta_{n}^{s}(x), s \geq 1$, which does not imply zero estimates at $x=0,1$.

Proof. The special results for $s=0,1,2$ are obvious. By the formulas on P. 14 of [11] we have $\bar{T}_{n 4}^{*}(x)=\bar{T}_{n 4}(x)=n^{-2} \delta_{n}^{2}(x)\left[3 n(n-2) \delta_{n}^{2}(x)+1\right] \leq 4 \delta_{n}^{2}(x) \Delta_{n}^{2}(x)$. By the Schwartz inequality, we have $\bar{T}_{n 3}^{*}(x) \leq\left[\bar{T}_{n 2}^{*}(x) \bar{T}_{n 4}^{*}(x)\right]^{1 / 2}=\delta_{n}(x)\left[\bar{T}_{n 4}^{*}(x)\right]^{1 / 2} \leq$ $2 \delta_{n}^{2}(x) \Delta_{n}(x)$. For $s \geq 4$, both $T_{n, 2 r}(x)$ and $T_{n, 2 r+1}(x)$ can be expressed as $n x(1-$ $x) \sum_{l=0}^{r-1}[n x(1-x)]^{l} Q_{r l}(x)$, where $Q_{r l}(x)$ are polynomials in $x$ with coefficients depending on $r$ and $l$ only [see Eq.5 of 32]. Similar to [10], this implies that $\bar{T}_{n, 2 r}^{*}(x)=\bar{T}_{n, 2 r}(x) \leq A_{2 r} \delta_{n}^{2}(x) \Delta_{n}^{2 r-2}(x)$. By Schwartz inequality again $\bar{T}_{n, 2 r+1}^{*}(x) \leq$ $\left[\bar{T}_{n 2}(x) \bar{T}_{n, 4 r}(x)\right]^{1 / 2} \leq A_{2 r+1} \delta_{n}^{2}(x) \Delta_{n}^{2 r-1}(x)$. The proof of the Lemma is complete.

\section{Appendix B. Proof of Lemma A.1}

Similar to [10], we want to prove that, for $r \geq 0$, there exist polynomials of the form

$$
Q_{\boldsymbol{m} r}^{f}(\boldsymbol{x})=\sum_{\boldsymbol{k}=\mathbf{0}}^{\boldsymbol{m}}\left\{f\left(\frac{\boldsymbol{k}}{\boldsymbol{m}}\right)+\sum_{i=2}^{r} \frac{1}{i !} \sum_{\langle\boldsymbol{i}\rangle=i}\left(\begin{array}{c}
\langle\boldsymbol{i}\rangle \\
\boldsymbol{i}
\end{array}\right) f^{(\boldsymbol{i})}\left(\frac{\boldsymbol{k}}{\boldsymbol{m}}\right) \prod_{j=1}^{d} \frac{1}{m_{j}^{i_{j}}} \tau_{r i_{j}}\left(x_{j}, m_{j}\right)\right\} p_{\boldsymbol{m}, \boldsymbol{k}}(\boldsymbol{x}),
$$

where $\left(\begin{array}{c}\langle\boldsymbol{i}\rangle \\ \boldsymbol{i}\end{array}\right)=\left(\begin{array}{c}\langle\boldsymbol{i}\rangle \\ i_{1}, \ldots, i_{d}\end{array}\right)$ is the multinomial coefficient, and $\tau_{r i}(x, m)$ 's are polynomials, independent of $f$, in $x$ of degree $i$, in $m$ of degree $\lfloor i / 2\rfloor$, such that for each function 
$f \in C^{(r)}[0,1]^{d}$,

$$
\left|f(\boldsymbol{x})-Q_{\boldsymbol{m} r}^{f}(\boldsymbol{x})\right| \leq C_{r, d}^{\prime} \omega^{(r)}\left[D_{\boldsymbol{m}}(\boldsymbol{x})\right] D_{\boldsymbol{m}}^{0 \vee(r-2)}(\boldsymbol{x})\left[\sum_{j=1}^{d} \delta_{m_{j}}\left(x_{j}\right)\right]^{2 \wedge r}
$$

with $C_{r, d}^{\prime}$ depending only on $r$ and $d$.

If $f \in C^{(r)}[0,1]^{d}, r \geq 1$, by the Taylor expansion of $f(\boldsymbol{k} / \boldsymbol{m})$ at $\boldsymbol{x}$, we have

$$
\begin{aligned}
f(\boldsymbol{x})= & f\left(\frac{\boldsymbol{k}}{\boldsymbol{m}}\right)-\sum_{i=1}^{r} \frac{1}{i !} \sum_{\langle\boldsymbol{i}\rangle=i}\left(\begin{array}{c}
\langle\boldsymbol{i}\rangle \\
\boldsymbol{i}
\end{array}\right) \prod_{j=1}^{d}\left(\frac{k_{j}}{m_{j}}-x_{j}\right)^{i_{j}} f^{(\boldsymbol{i})}(\boldsymbol{x}) \\
& +\frac{1}{r !}\left\{\sum_{\langle\boldsymbol{i}\rangle=r}\left(\begin{array}{c}
r \\
\boldsymbol{i}
\end{array}\right) \prod_{j=1}^{d}\left(\frac{k_{j}}{m_{j}}-x_{j}\right)^{i_{j}}\left[f^{(\boldsymbol{i})}(\boldsymbol{x})-f^{(\boldsymbol{i})}\left(\boldsymbol{\xi}_{\boldsymbol{k}}^{(r)}\right)\right]\right\},
\end{aligned}
$$

where $\boldsymbol{\xi}_{\boldsymbol{k}}^{(r)}$ is on the line segment connecting $\boldsymbol{x}$ and $\boldsymbol{k} / \boldsymbol{m}$. This equation is also true when $r=0$ by defining $\boldsymbol{\xi}_{\boldsymbol{k}}^{(0)}=\boldsymbol{k} / \boldsymbol{m}$ and the empty sum to be zero. Multiplying both sides by $p_{\boldsymbol{m}, \boldsymbol{k}}(\boldsymbol{x})$ and taking summation over $\mathbf{0} \leq \boldsymbol{k} \leq \boldsymbol{m}$, we obtain

$$
f(\boldsymbol{x})=B^{f}(\boldsymbol{x})-\sum_{i=2}^{r} \frac{1}{i !} \sum_{\langle\boldsymbol{i}\rangle=i}\left(\begin{array}{c}
\langle\boldsymbol{i}\rangle \\
\boldsymbol{i}
\end{array}\right) \prod_{j=1}^{d} \bar{T}_{m_{j} i_{j}}\left(x_{j}\right) f^{(\boldsymbol{i})}(\boldsymbol{x})+R_{m}^{(r)}(\boldsymbol{x}),
$$

where $r \geq 0$, empty sum is zero, and

$$
R_{m}^{(r)}(\boldsymbol{x})=\frac{1}{r !}\left\{\sum_{\langle\boldsymbol{i}\rangle=r}\left(\begin{array}{l}
r \\
\boldsymbol{i}
\end{array}\right) \sum_{\boldsymbol{k}=0}^{\boldsymbol{m}} \prod_{j=1}^{d} \frac{1}{m_{j}^{i_{j}}}\left(k_{j}-m_{j} x_{j}\right)^{i_{j}} p_{m_{j} k_{j}}\left(x_{j}\right)\left[f^{(\boldsymbol{i})}(\boldsymbol{x})-f^{(\boldsymbol{i})}\left(\boldsymbol{\xi}_{\boldsymbol{k}}^{(r)}\right)\right]\right\}
$$

For each $\delta>0$, define $\lambda=\lambda(\boldsymbol{x}, \boldsymbol{y} ; \delta)=\lfloor|\boldsymbol{x}-\boldsymbol{y}| / \delta\rfloor$, where $\lfloor x\rfloor$ is the integer part of $x \geq 0$. Then $\lambda \delta \leq|\boldsymbol{x}-\boldsymbol{y}|<(\lambda+1) \delta$, and for $g \in C[0,1]^{d},|g(\boldsymbol{x})-g(\boldsymbol{y})| \leq(\lambda+1) \omega(g, \delta)$.

If $f \in C^{(r)}[0,1]^{d}, r=0,1$, then similar to the proofs of Theorems 1.6.1 and 1.6.2 of [11, pp. 20- 21] and by $\mathrm{B} 3$ we have $\left|f(\boldsymbol{x})-B_{\boldsymbol{m}}^{f}(\boldsymbol{x})\right|=\left|R_{m}^{(r)}(\boldsymbol{x})\right|$. Because 
$\lambda(\boldsymbol{x}, \boldsymbol{k} / \boldsymbol{m} ; \delta) \leq \delta^{-1} \sum_{j=1}^{d}\left|k_{j}-m_{j} x_{j}\right| / m_{j}$, by Lemma A.2 with $s=0,1,2$, we have

$$
\begin{aligned}
\left|f(\boldsymbol{x})-B_{\boldsymbol{m}}^{f}(\boldsymbol{x})\right| & \leq \sum_{\langle\boldsymbol{i}\rangle=r} \omega_{\boldsymbol{i}}(\delta)\left[\prod_{j=1}^{d} \bar{T}_{m_{j}, i_{j}}^{*}\left(x_{j}\right)+\frac{1}{\delta} \sum_{l=1}^{d} \bar{T}_{m_{l}, i_{l}+1}^{*}\left(x_{l}\right) \prod_{\substack{1 \leq j \leq d \\
j \neq l}} \bar{T}_{m_{j}, i_{j}}^{*}\left(x_{j}\right)\right] \\
& \leq \sum_{\langle\boldsymbol{i}\rangle=r} \omega_{\boldsymbol{i}}(\delta)\left[\prod_{j=1}^{d} \delta_{m_{j}}^{i_{j}}\left(x_{j}\right)+\frac{1}{\delta} \sum_{l=1}^{d} \delta_{m_{l}}^{i_{l}+1}\left(x_{l}\right) \prod_{\substack{1 \leq j \leq d \\
j \neq l}} \delta_{m_{j}}^{i_{j}}\left(x_{j}\right)\right] .
\end{aligned}
$$

The estimates in $\mathrm{A} 2$ follow from $\mathrm{B} 4$ with $\delta=\max _{1 \leq j \leq d} \delta_{m_{j}}\left(x_{j}\right)$. This also proves B2 with $r=0,1$ and $Q_{m r}^{f}=B^{f}$. So part (i) is proved.

If $r \geq 2$, then we have

$$
\begin{aligned}
\left|R_{\boldsymbol{m}}^{(r)}(\boldsymbol{x})\right| \leq & \frac{1}{r !}\left\{\sum_{\langle\boldsymbol{i}\rangle=r}\left(\begin{array}{c}
r \\
\boldsymbol{i}
\end{array}\right) \omega_{\boldsymbol{i}}(\delta)\left[\prod_{j=1}^{d} \bar{T}_{m_{j} i_{j}}^{*}\left(x_{j}\right)+\frac{1}{\delta} \sum_{l=1}^{d} \bar{T}_{m_{l}, i_{l}+1}^{*}\left(x_{l}\right) \prod_{\substack{1 \leq j \leq d \\
j \neq l}} \bar{T}_{m_{j} i_{j}}^{*}\left(x_{j}\right)\right]\right\} \\
\leq & \frac{1}{r !}\left\{\sum _ { \langle i \rangle = r } ( \begin{array} { c } 
{ r } \\
{ \boldsymbol { i } }
\end{array} ) \omega _ { \boldsymbol { i } } ( \delta ) \left[\prod_{j=1}^{d} A_{i_{j}} \delta_{m_{j}}^{2 \wedge i_{j}}\left(x_{j}\right) \Delta_{m_{j}}^{0 \vee\left(i_{j}-2\right)}\left(x_{j}\right)\right.\right. \\
& \left.\left.+\frac{1}{\delta} \sum_{l=1}^{d} A_{i_{l}+1} \delta_{m_{l}}^{2 \wedge\left(i_{l}+1\right)}\left(x_{l}\right) \Delta_{m_{l}}^{0 \vee\left(i_{l}-1\right)}\left(x_{l}\right) \prod_{\substack{1 \leq j \leq d \\
j \neq l}} A_{i_{j}} \delta_{m_{j}}^{2 \wedge i_{j}}\left(x_{j}\right) \Delta_{m_{j}}^{0 \vee\left(i_{j}-2\right)}\left(x_{j}\right)\right]\right\} .
\end{aligned}
$$

Choosing $\delta=D_{\boldsymbol{m}}(\boldsymbol{x})$, we have

$$
\begin{aligned}
\left|R_{\boldsymbol{m}}^{(r)}(\boldsymbol{x})\right| \leq & \omega^{(r)}(\delta) \frac{1}{r !}\left\{\sum_{\langle\boldsymbol{i}\rangle=r}\left(\begin{array}{c}
r \\
\boldsymbol{i}
\end{array}\right) \prod_{j=1}^{d} A_{i_{j}} \delta_{m_{j}}^{2 \wedge i_{j}}\left(x_{j}\right) \Delta_{m_{j}}^{0 \vee\left(i_{j}-2\right)}\left(x_{j}\right)\right. \\
& \left.+\sum_{l=1}^{d} \sum_{\langle\boldsymbol{i}\rangle=r}\left(\begin{array}{c}
r \\
\boldsymbol{i}
\end{array}\right) A_{i_{l}+1} \delta_{m_{l}}^{2 \wedge i_{l}}\left(x_{l}\right) \Delta_{m_{l}}^{0 \vee\left(i_{l}-2\right)}\left(x_{l}\right) \prod_{\substack{1 \leq j \leq d \\
j \neq l}} A_{i_{j}} \delta_{m_{j}}^{2 \wedge i_{j}}\left(x_{j}\right) \Delta_{m_{j}}^{0 \vee\left(i_{j}-2\right)}\left(x_{j}\right)\right\} \\
\leq & C(r, d) \omega^{(r)}(\delta) \max _{1 \leq j \leq d} \Delta_{m_{j}}^{r-2}\left(x_{j}\right)\left[\sum_{j=1}^{d} \delta_{m_{j}}\left(x_{j}\right)\right]^{2} .
\end{aligned}
$$

Similar to [10] we shall prove the existence of $Q_{m r}^{f}$ by induction in $r$. Assuming that all $Q_{m i}^{f}$ for $i<r$ are established, we iteratively define

$$
Q_{\boldsymbol{m} r}^{f}(\boldsymbol{x})=B^{f}(\boldsymbol{x})-\sum_{i=2}^{r} \frac{1}{i !} \sum_{\langle\boldsymbol{i}\rangle=i}\left(\begin{array}{c}
\langle\boldsymbol{i}\rangle \\
\boldsymbol{i}
\end{array}\right) \prod_{j=1}^{d} \bar{T}_{m_{j} i_{j}}\left(x_{j}\right) Q_{\boldsymbol{m}, r-i}^{f^{(i)}}(\boldsymbol{x}) .
$$


By Lemma A.2 and the inductive assumption, $\mathrm{B} 2$ is satisfied by $\mathrm{B} 5$ as following.

$$
\begin{aligned}
\left|f(\boldsymbol{x})-Q_{\boldsymbol{m} r}^{f}(\boldsymbol{x})\right| \leq & \sum_{i=2}^{r} \frac{1}{i !} \sum_{\langle\boldsymbol{i}\rangle=i}\left(\begin{array}{c}
\langle\boldsymbol{i}\rangle \\
\boldsymbol{i}
\end{array}\right) \prod_{j=1}^{d} \bar{T}_{m_{j} i_{j}}^{*}\left(x_{j}\right)\left|f^{(i)}(\boldsymbol{x})-Q_{\boldsymbol{m}, r-i}^{f^{(i)}}(\boldsymbol{x})\right|+\left|R_{\boldsymbol{m}}^{(r)}(\boldsymbol{x})\right| \\
\leq & \sum_{i=2}^{r} \frac{C_{r, d}^{\prime \prime}}{i !} D_{\boldsymbol{m}}(\boldsymbol{x})^{i-2}(\boldsymbol{x})\left[\sum_{j=1}^{d} \delta_{m_{j}}\left(x_{j}\right)\right]^{2} \\
& \cdot \omega^{(r)}\left[D_{\boldsymbol{m}}(\boldsymbol{x})\right] D_{\boldsymbol{m}}^{0 \vee(r-i-2)}(\boldsymbol{x})\left[\sum_{j=1}^{d} \delta_{m_{j}}\left(x_{j}\right)\right]^{2 \wedge(r-i)}+\left|R_{\boldsymbol{m}}^{(r)}(\boldsymbol{x})\right| \\
\leq & C_{r, d}^{\prime \prime \prime} \omega^{(r)}\left[D_{\boldsymbol{m}}(\boldsymbol{x})\right] D_{\boldsymbol{m}}^{0 \vee(r-2)}(\boldsymbol{x})\left[\sum_{j=1}^{d} \delta_{m_{j}}\left(x_{j}\right)\right]^{2} .
\end{aligned}
$$

Since $f(\boldsymbol{x}) \geq \delta>0$, by an obvious generalization of remark (a) on p. 241 of [10] with $\boldsymbol{h}=1 / \boldsymbol{m}$ we know that $P_{\boldsymbol{m}+r}(\boldsymbol{x})=Q_{\boldsymbol{m} r}^{f}(\boldsymbol{x})$ is a $d$-variate polynomial of degree $\boldsymbol{m}+r=\left(m_{1}+r, \ldots, m_{d}+r\right)$ with positive coefficients for all $\boldsymbol{m} \geq \boldsymbol{m}_{r}\left(m, \mathscr{M}_{r}\right)$ so that

$$
\left|f(\boldsymbol{x})-P_{\boldsymbol{m}+r}(\boldsymbol{x})\right| \leq C_{r, d} \omega^{(r)}\left[D_{\boldsymbol{m}}(\boldsymbol{x})\right] D_{\boldsymbol{m}}^{r-2}(\boldsymbol{x})\left[\sum_{j=1}^{d} \delta_{m_{j}}\left(x_{j}\right)\right]^{2}
$$

Then A3 follows for all $\boldsymbol{m}$ and a larger $C_{r, d}$ from $\Delta_{m_{j}}=\mathcal{O}\left(\Delta_{m_{j}+r}\right)$ for all $r \geq 2$. The proof of part (ii) is complete.

We omit the proof of part (iii) because it is almost the same as that of Lemma 3.1 below.

\section{Appendix C. Proof of Lemma 3.1}

By parts (i) and (ii) of Lemma A.1 we have $f_{0}(\boldsymbol{t})-P_{\boldsymbol{m}}(\boldsymbol{t})=R_{\boldsymbol{m}}(\boldsymbol{t})$, where $P_{\boldsymbol{m}}(\boldsymbol{t})$ is a polynomial with positive coefficients and $R_{\boldsymbol{m}}(\boldsymbol{t})$ satisfies $\left|R_{\boldsymbol{m}}(\boldsymbol{t})\right| \leq C_{0}(d, f) m_{0}^{-(r+\alpha) / 2}$, $0 \leq \boldsymbol{t} \leq 1$. So $f(\boldsymbol{t})-Q_{\tilde{\boldsymbol{m}}}(\boldsymbol{t})=R_{\tilde{\boldsymbol{m}}}(\boldsymbol{t})$, where $Q_{\tilde{\boldsymbol{m}}}(\boldsymbol{t})=P_{\boldsymbol{m}}(\boldsymbol{t}) \prod_{i=1}^{d} t_{i}^{a_{i}}\left(1-t_{i}\right)^{b_{i}}=$ $\sum_{\boldsymbol{i}=0}^{\tilde{\boldsymbol{m}}} a(\boldsymbol{i}) \cdot \beta_{\tilde{\boldsymbol{m}} \boldsymbol{i}}(\boldsymbol{t})$ is a polynomial of degree $\tilde{\boldsymbol{m}}=\boldsymbol{m}+\boldsymbol{a}+\boldsymbol{b}$ with positive coefficients, $R_{\tilde{\boldsymbol{m}}}(\boldsymbol{t})=R_{\boldsymbol{m}}(\boldsymbol{t}) \prod_{i=1}^{d} t_{i}^{a_{i}}\left(1-t_{i}\right)^{b_{i}}$, and $\left|R_{\tilde{\boldsymbol{m}}}(\boldsymbol{t})\right| \leq C_{0}(d, f) m_{0}^{-(r+\alpha) / 2}, 0 \leq \boldsymbol{t} \leq 1$. For large $\boldsymbol{m}, \rho_{\tilde{\boldsymbol{m}}}:=\int_{[0,1]^{d}} R_{\tilde{\boldsymbol{m}}}(\boldsymbol{t}) d \boldsymbol{t} \leq C_{0}(d, f) m_{0}^{-(r+\alpha) / 2}<c_{0}<1$. Since $f(\boldsymbol{t})$ and 
$\beta_{\tilde{\boldsymbol{m}} \boldsymbol{i}}(\boldsymbol{t})$ are densities on $[0,1]^{s}, \sum_{\boldsymbol{i}=0}^{\tilde{\boldsymbol{m}}} a(\boldsymbol{i})=1-\rho_{\tilde{\boldsymbol{m}}}>0$. So normalizing $a(\boldsymbol{i})$ we obtain $f_{\tilde{\boldsymbol{m}}}\left(\boldsymbol{t} ; \boldsymbol{p}_{0}\right)=Q_{\tilde{\boldsymbol{m}}}(\boldsymbol{t}) /\left(1-\rho_{\tilde{\boldsymbol{m}}}\right)=\sum_{\boldsymbol{i}=0}^{\tilde{\boldsymbol{m}}} p_{0}(\boldsymbol{i}) \cdot \beta_{\tilde{\boldsymbol{m}} \boldsymbol{i}}(\boldsymbol{t})$, where $p_{0}(\boldsymbol{i})=a(\boldsymbol{i}) /\left(1-\rho_{\tilde{\boldsymbol{m}}}\right)$.

Noticing that $f_{0}(\boldsymbol{t}) \geq \delta_{0}>0$, we have

$$
\begin{aligned}
\left|f_{\tilde{\boldsymbol{m}}}\left(\boldsymbol{t} ; \boldsymbol{p}_{0}\right)-f(\boldsymbol{t})\right| / f(\boldsymbol{t}) & =\left(1-\rho_{\tilde{\boldsymbol{m}}}\right)^{-1}\left|R_{\tilde{\boldsymbol{m}}}(\boldsymbol{t}) / f(\boldsymbol{t})+\rho_{\tilde{\boldsymbol{m}}}\right| \\
& =\left(1-\rho_{\tilde{\boldsymbol{m}}}\right)^{-1}\left|R_{\boldsymbol{m}}(\boldsymbol{t}) / f_{0}(\boldsymbol{t})+\rho_{\tilde{\boldsymbol{m}}}\right| \\
& \leq\left(1-c_{0}\right)^{-1} C_{0}(d, f)\left(1 / \delta_{0}+1\right) m_{0}^{-(r+\alpha) / 2}
\end{aligned}
$$

The proof is complete.

\section{Appendix D. Proof of Theorem 3.2}

The approximate Bernstein log likelihood is $\ell\left(f_{\boldsymbol{m}}\right)=\ell(\boldsymbol{p})=\sum_{i=1}^{n} \log \left[f_{\boldsymbol{m}}\left(\boldsymbol{x}_{i} ; \boldsymbol{p}\right)\right]$. Define the log-likelihood ratio $\mathcal{R}(\boldsymbol{p})=\ell(f)-\ell(\boldsymbol{p})$, where $\ell(f)=\sum_{i=1}^{n} \log f\left(\boldsymbol{x}_{i}\right)$. For an $\epsilon_{0} \in(0,1)$, we define subset $\mathcal{A}_{\boldsymbol{m}}\left(\epsilon_{0}\right)$ of $\mathbb{S}_{\boldsymbol{m}}$ so that, for all $\boldsymbol{x} \in[0,1]^{d}, \mid f_{\boldsymbol{m}}(\boldsymbol{x} ; \boldsymbol{p})-$ $f(\boldsymbol{x}) \mid / f(\boldsymbol{x}) \leq \epsilon_{0}<1$. Clearly, such an $\mathcal{A}_{\boldsymbol{m}}\left(\epsilon_{0}\right)$ is nonempty since $\boldsymbol{p}_{0} \in \mathcal{A}_{\boldsymbol{m}}\left(\epsilon_{0}\right)$. By Taylor expansion we have, for all $\boldsymbol{p} \in \mathcal{A}_{\boldsymbol{m}}\left(\epsilon_{0}\right)$,

$$
\mathcal{R}(\boldsymbol{p})=-\sum_{i=1}^{n}\left[Z_{i}(\boldsymbol{p})-\frac{1}{2} Z_{i}^{2}(\boldsymbol{p})\right]+\mathcal{O}\left(R_{\boldsymbol{m} n}(\boldsymbol{p})\right) \quad \text { a.s. }
$$

where $R_{\boldsymbol{m} n}(\boldsymbol{p})=\sum_{i=1}^{n} Z_{i}^{2}(\boldsymbol{p})$, and $Z_{i}(\boldsymbol{p})=\left[f_{\boldsymbol{m}}\left(\boldsymbol{x}_{i} ; \boldsymbol{p}\right)-f\left(\boldsymbol{x}_{i}\right)\right] / f\left(\boldsymbol{x}_{i}\right), i=1, \ldots, n$. Since $\mathrm{E}\left[Z_{i}(\boldsymbol{p})\right]=0, \sigma^{2}\left[Z_{i}(\boldsymbol{p})\right]=\mathrm{E}\left[Z_{i}^{2}(\boldsymbol{p})\right]=D^{2}(\boldsymbol{p})$, by the law of iterated logarithm we have

$$
\sum_{i=1}^{n} Z_{i}(\boldsymbol{p}) / \sigma\left[Z_{i}(\boldsymbol{p})\right]=\mathcal{O}(\sqrt{n \log \log n}) \quad \text { a.s. }
$$

By the strong law of large numbers we have

$$
\mathcal{R}(\boldsymbol{p})=\frac{n}{2} D^{2}(\boldsymbol{p})-\mathcal{O}(D(\boldsymbol{p}) \sqrt{n \log \log n})+\mathcal{O}\left(n D^{2}(\boldsymbol{p})\right) \quad \text { a.s. }
$$


If $D^{2}(\boldsymbol{p})=r_{n}^{2}=\log n / n$, then, by (D1), there is an $\eta>0$ such that $\mathcal{R}(\boldsymbol{p}) \geq \eta \log n$, a.s. At $\boldsymbol{p}=\boldsymbol{p}_{0}$, if $m_{0}=C n^{1 / k}$ then by assumption (A.1) we have $D^{2}\left(\boldsymbol{p}_{0}\right)=\mathcal{O}\left(m_{0}^{-k}\right)=$ $\mathcal{O}\left(n^{-1}\right)$. By D1 again we have $\mathcal{R}\left(\boldsymbol{p}_{0}\right)=\mathcal{O}(\sqrt{\log \log n})$ a.s.. Therefore, similar to the proof of Lemma 1 of [33], we have

$$
D^{2}(\hat{\boldsymbol{p}})=\int_{[0,1]^{d}} \frac{\left[f_{\boldsymbol{m}}(\boldsymbol{x} ; \hat{\boldsymbol{p}})-f(\boldsymbol{x})\right]^{2}}{f(\boldsymbol{x})} d \boldsymbol{x}<\frac{\log n}{n} \quad \text { a.s. }
$$

The inequality (8) follows immediately. Based on (8) and the boundedness of $f$, the inequality (9) is obtained. 